\title{
Automatic Screening of Lung Diseases by 3D Active Contour Method for Inhomogeneous Motion Estimation in CT Image Pairs
}

\author{
Pikul VEJJANUGRAHA ${ }^{1,2, *}$, Kazunori KOTANI ${ }^{1}$, \\ Waree KONGPRAWECHNON ${ }^{2}$, Toshiaki KONDO ${ }^{2}$ and \\ Kanokvate TUNGPIMOLRUT ${ }^{3}$
}

${ }^{1}$ School of Information Science, Japan Advanced Institute of Science and Technology, Ishikawa, Japan ${ }^{2}$ School of Information, Communication and Computer Technologies, Sirindhorn International Institute of Technology, Pathumthani 12120, Thailand

${ }^{3}$ The National Electronics and Computer Technology Center (NECTEC), The National Science and Technology Development Agency, Pathumthani 12120, Thailand

('Corresponding author's e-mail: pikulvej@gmail.com)

Received: 8 June 2020, Revised: 1 October 2020, Accepted: 7 October 2020

\begin{abstract}
Lung diseases are now the third leading cause of death worldwide because of the many risk factors we are exposed to daily, such as air pollution, tobacco use, viruses (such as COVID-19), and bacteria. This work introduces a new approach of the 3D Active Contour Model (3D ACM) to estimate an inhomogeneous motion of lungs, which can be used to analyze lung disease patterns using a hierarchical predictive model. The biophysical model of lungs consists of End Expiratory (EE) and End Inspiratory (EI) models, generated by high-resolution computed tomography images (HRCT). A proposed technique uses the $3 \mathrm{D} \mathrm{ACM}$ to estimate the velocity vector by using the corresponding points on the parametric surface model of the EE model to the EI model. The external energy from the EI models is the external force that pushes the 3D parametric surface to reach the boundary. The external forces, such as the balloon force and Gradient Vector Flow (GVF), were adjusted adaptively based on the $Z_{\text {ratio }}$ which was calculated from the ratio of the maximum value of EI to EE on the $Z$ axis. Next, the feature representation is studied and evaluated based on the lung structure, separated into five lobes. The stepwise regression, Support Vector Machine (SVM), and Artificial Neural Network (ANN) techniques are applied to classify the lung diseases into normal, obstructive lung, and restrictive lung diseases. In conclusion, the inhomogeneous motion pattern of lungs integrated with medical-based knowledge can be used to analyze lung diseases by differentiating normal and abnormal motion patterns and separating restrictive and obstructive lung diseases.
\end{abstract}

Keywords: 3D active contour model, lung disease analysis, inhomogeneous motion pattern, velocity vector map, hierarchical classification

\section{Introduction}

A lung is a vital and heterogeneous organ in the human body. An abnormality of respiration leads to regional differences, depending on diverse functions in the lymphatic system, immune system, metabolic system, and mechanical properties such as gravity. According to the World Health Organization (WHO) [1], lung diseases, especially Chronic Obstructive Pulmonary Disease (COPD), are the third leading cause of death globally in 2016. Tobacco use is also the fourth risk factor of death in Thailand. It was found that the death rate from impaired lungs is increased by the primary risk factors, which are tobacco use, air 
pollution, chemicals, viruses, and bacteria. The lower respiratory infection and trachea, bronchus, and lung cancer also include the top 10 global causes of death. The cause factors directly affect our daily life nowadays. The early detection of respiratory abnormality is essential to consider when it is reversible or almost fully reversible. The predominant diseases in each regional lobe can be divided into three regions: upper lobe [2,3], middle lobe [4], and lower lobe [3-5]. Disease characterization is challenging because of its diversity in many aspects. It can be considered by lung components such as airways, air sacs, interstitium, blood vessels, pleura, and chest wall. Initially, to diagnose a lung disease is to distinguish an obstructive lung disease and a restrictive lung disease. On the physical level, a patient with obstructive lung disease has difficulty during expiration because of the narrowed or blocked airways (airflow limitation). The remaining air inside affects the residual volume (remaining high) of the lung and leads to air trapping and hyperinflation problems, which can be observed in the anterior-posterior (AP) axis of CT images. In contrast, a restrictive lung disease limits the ability to inhale air. The patient cannot take a deep breath, which affects the total lung capacity and residual volume (low).

The criteria to diagnose obstructive and restrictive lung diseases are defined by the Pulmonary Function Tests (PFTs). The PFTs consist of three main parameters: 1) The Forced Vital Capacity (FVC) test shows the amount of air that a person can quickly and forcefully breathe out after a deep breath, 2) the Forced Expiratory Volume in One Second (FEV1) test shows the amount of air a person can forcefully exhale in one second of the FVC test, and 3) the Total Lung Capacity (TLC) test describes the volume of air remaining in the lung after exhalation. The FEV1/FVC ratio is used to diagnose the type of lung disease and the severity of a disease. The $\mathrm{FEV}_{1} / \mathrm{FVC}$ ratio is decreased in the obstructive pattern and increased in the restrictive pattern. For example, an $\mathrm{FEV}_{1} / \mathrm{FVC}$ ratio of less than 0.7 [18] is considered as COPD and the stage of COPD is classified by the percent of $\mathrm{FEV}_{1}: \mathrm{Mild} \mathrm{FEV}_{1} \geq 80 \%$, Moderate $50 \% \leq$ $\mathrm{FEV}_{1}<80 \%$, Severe $30 \% \leq \mathrm{FEV}_{1}<50 \%$, and Very Severe $\mathrm{FEV}_{1}<30 \%$. These tests are the gold standard to diagnose COPD [6,7]. The TLC increased in a normal, obstructive pattern by the remaining air in the lungs and decreased in a restrictive pattern. These characteristics of lung patterns are detected and analyzed to diagnose lung diseases. However, to identify a complex lung disease, thorough lung function testing is required, such as an X-ray and CT scan. In this work, the Deep Inspiration Breath-Hold (DIBH) technique of CT-scan is used to characterize two different phases of respiration which are the End Inspiratory Phase (EI-images) and End Expiratory Phase (EE-images).

Nowadays, radiologists have some difficulties analyzing the two images (EI and EE images) at one time. They need to examine EI and EE images on two monitors separately and manually to locate the anatomical reference points between the two models, such as blood vessels, spinal cord (Thoracic Vertebrae), and trachea, to diagnose diseases. This requires experience to detect the abnormalities for answering the clinical questions on a diagnostic test. Therefore, to interpret the medical information from the two phases of respiratory CT images (EI and EE images) is still a research gap at present.

This research introduces a new approach to determining the lungs' velocity vector map from paired inspiratory-expiratory chest CT images from the 3D Active Contour Model (3D ACM) technique. 3D ACMs are frequently used in medical image analysis because they can detect a non-rigid object by using the parametric curve, and the parametric curve drives by the potential forces from the boundary of the target object. In this work, the ACM is used to overcome the non-rigid registration of the deformation surface, and its external force is also useful to help estimate the motion of the lung. The computing time can be reduced by using the ACM. It was found that the Finite Element technique also provides reliable results $[8,10]$, but it took $88.6 \mathrm{~h}$ to analyze images [8]. In contrast, the computation time can be reduced by using the 3D ACM. For example, this work took approximately $10 \mathrm{~min}$.

Because the shape of the lungs is non-rigid and individual for each person, an inhomogeneous pattern of the lung motion is considered for screening the lung diseases by two modules. Firstly, the normal and abnormal lungs are classified. The weighted average precision is $85.6 \%$, with 7 False Negative (FN) cases. Secondly, restrictive and obstructive patterns are classified. From the integration of lung pathology and anatomy knowledge, the inhomogeneous model of lung lobes can predict the predominance of lung diseases with $82.34 \%$ weighted average precision with $8 \mathrm{FN}$ cases. A comparison between the ANN and SVM techniques shows that SVM has the higher performance to maximize the 
http://wjst.wu.ac.th

support vectors from 2 groups of data. Therefore, it is concluded that lung motions can be used to prescreen lung diseases.

This paper is organized as follows. The Materials and Methods section provides the necessary information about data collection and experimental settings and also describes the methodology. The next section describes an evaluation technique and shows the results. Then, the output is interpreted in the Discussion section. Finally, the summary and future works are concluded in the last section.

\section{Materials and methods}

The methodology of the proposed technique is demonstrated in Figure 1. It consisted of 6 main steps: the data acquisition, preprocessing, 3D surface rendering, 3D parametric Active Contour Model, velocity vector map and lung lobe separation, and feature analysis and classification.

\section{Data acquisition}

The CT images and medical advice were obtained from the Thammasat University Hospital, Pathum Thani, Thailand. The input format is EE and EI high-resolution computed tomography (HRCT) images in the transverse plane with $1 \mathrm{~mm}$ thickness (200 images per image stack) or $2 \mathrm{~mm}$ thickness (100 images per image stack). The HRCT images are compressed in DICOM format. The 2-D resolution of CT images is $512 \times 512$ pixels with an $(i, j, k)$ coordinate system. Compared with the cartesian coordinate system, the axial plane is the Z-axis, coronal plane is the Y-axis, and sagittal plane is the $\mathrm{X}$-axis. The interpretation of HRCT images is done by relative quantitative measurement of radio density by adjusting the intensity level of a 16-bit of gray-scale called Hounsfield Unit (HU). The window width (WW) and window level (WL) are the thresholds for setting the gray-scale range. A suitable WW and WL for the pulmonary images are 1000 and -700 [6,11], respectively. OsiriX MD software is used to render the 3D surface model of lungs. The EI and EE HRCT images are divided into left and right models, named as $\mathrm{EI}_{\text {right }}, \mathrm{EE}_{\text {right }}, \mathrm{EI}_{\text {left }}$, and $\mathrm{EE}_{\text {left. }}$

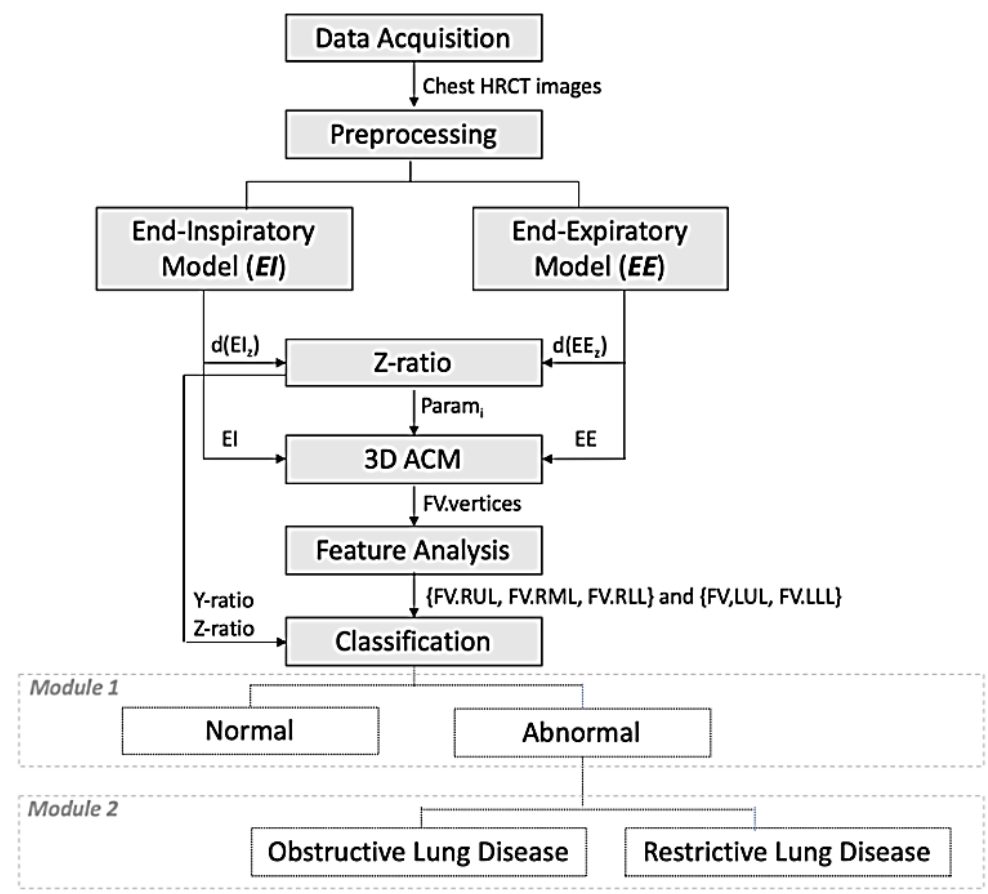

Figure 1 The overview of the purposed technique. 


\section{Preprocessing}

Image preparation, such as noise reduction and smoothening of surfaces, is performed to improve the segmentation efficiency and reduce unwanted noise. The noise from the small bronchi needs to be removed before rendering the 3D surface model. First, the WW and WL are set to show the pulmonary elements clearly. Then, the inverted filter is used to convert the intensity from -1024 to 1024 and from 1024 to -1024 . Then, the region of interest (ROI) is applied to the pulmonary area, and the intensity inside the ROI is converted into 1024, as demonstrated in Figure 2 (right).
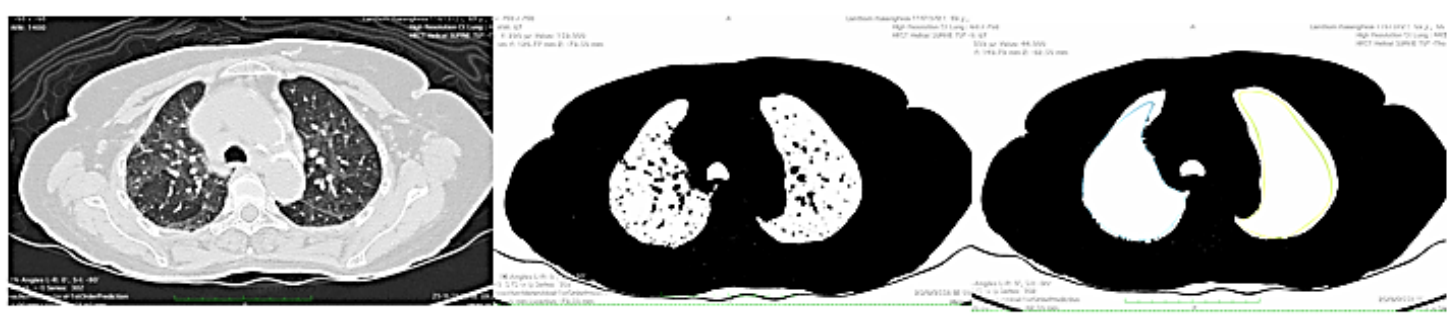

Figure 2 Noise reduction process: (left) WW and WL setting, (middle) invert filter, and (right) removing noise inside the lung.

\section{D Surface rendering}

3D Surface rendering is performed on the stack of CT images. The left and the right lungs are performed separately. Some unwanted areas, such as the primary airway, are removed. Then, 3D surface generation is applied to each lung model. Finally, four lung models are generated: $\mathrm{EI}_{\mathrm{right}}, \mathrm{EE}_{\mathrm{right}}, \mathrm{EI}_{\text {left }}$, and $\mathrm{EE}_{\text {left }}$ as shown in Figure 3. It can be observed that the central airway is already removed. According to computational time, resampling process is applied to the lung models to reduce the resolution to 20,000 vertices. Works in the literature $[9,12]$ show that 20,000 vertices are acceptable to estimate the motion of a lung with high accuracy.

Table 1 The location of landmark points.

\begin{tabular}{lll}
\hline Fissures & Landmark points $(\boldsymbol{x}, \boldsymbol{y}, \boldsymbol{z})$ & Anatomical location \\
\hline \multirow{4}{*}{$\mathrm{ROF}$} & $\mathrm{R}_{\mathrm{OF} 1}$ & Spinal cord: T3 \\
\cline { 2 - 3 } & $\mathrm{R}_{\mathrm{OF} 2}$ & $6^{\text {Th }}$ rib at midclavicular line \\
\cline { 2 - 3 } & $\mathrm{R}_{\mathrm{OF} 3}$ & $6^{\text {Th }}$ rib at midclavicular line \\
\hline \multirow{3}{*}{$\mathrm{RHF}$} & $\mathrm{R}_{\mathrm{HF} 1}$ & $4^{\text {Th }}$ rib \\
\cline { 2 - 3 } & $\mathrm{R}_{\mathrm{HF} 2}$ & $5^{\text {Th }}$ rib \\
\cline { 2 - 3 } & $\mathrm{R}_{\mathrm{HF} 3}$ & $5^{\text {Th }}$ rib \\
\hline \multirow{3}{*}{ LOF } & $\mathrm{L}_{\mathrm{OF} 1}$ & Spinal cord: T3 \\
\cline { 2 - 3 } & $\mathrm{L}_{\mathrm{OF} 2}$ & $6^{\text {Th }}$ rib at midclavicular line \\
\cline { 2 - 3 } & $\mathrm{L}_{\mathrm{OF} 3}$ & $6^{\text {Th }}$ rib at midclavicular line \\
\hline
\end{tabular}


http://wjst.wu.ac.th

Next, the landmark points are detected based on the anatomical location in order to generate an oblique fissure plane $(\boldsymbol{a x}+\boldsymbol{b} \boldsymbol{y}+\boldsymbol{c z}+\boldsymbol{d}=\mathbf{0})$ from 3 landmark points. An oblique fissure plane is used to separate the lung into 5 lobes: 2 lobes for the left lung and 3 lobes for the right lung. The landmark points are kept into 3 sets for each oblique fissure: Right Oblique fissure ROF $\left(\mathrm{R}_{\mathrm{OF} 1}, \mathrm{R}_{\mathrm{OF} 2}, \mathrm{R}_{\mathrm{OF} 3}\right)$, Right Horizontal fissure RHF $\left(\mathrm{R}_{\mathrm{HF} 1}, \mathrm{R}_{\mathrm{HF} 2}, \mathrm{R}_{\mathrm{HF} 3}\right)$, and Left oblique fissure LOF ( $\left.\mathrm{L}_{\mathrm{OF} 1}, \mathrm{~L}_{\mathrm{OF} 2}, \mathrm{~L}_{\mathrm{OF} 3}\right)$. The thoracic landmarks of ROF, RHF, and LOF are represented in Figure 3 and are based on the anatomical location, as described in Table 1.
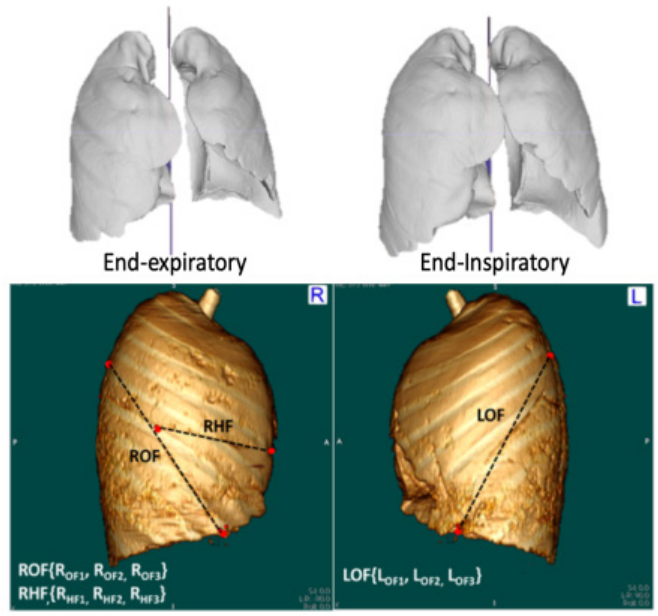

Figure $3 \mathrm{EE}$ and EI models and representation of 3 fissures on the 3D model: right lung (ROF and RHF), left lung (LOF).

\section{D Active Contour Models (3D ACMs)}

The 3D ACMs are generally used as a non-rigid segmentation-based technique, especially in biomedical images. The concept of 3D ACM is to minimize the internal and external energies, and the desired boundary of the target will stop the final evaluation of the active contour. In this study, the velocity vector map of the respiratory motion starting from the EE to the EI phase is detected by estimating the control point on the 3D ACM technique. The apex part of the lung is detected and assigned as the reference point for aligning the model. The parameters of the 3D ACM for adjusting internal and external forces are set due to the sensitivity to the shape, such as a sharp edge. Therefore, they are adaptable depending on the which represents the shape of the lungs.

The 3D Parametric Active Contour Model is performed to estimate the velocity vector between the EE and EI lung models. A mesh represents the control points of the $3 \mathrm{D}$ active contour. The energy function in 3D ACM develops the parameter function to control the control points in more dimensions as $\boldsymbol{v}:[\mathbf{0}, \mathbf{1}] \times[\mathbf{0}, \mathbf{1}] \rightarrow \mathbb{R}^{3}$. The $3 \mathrm{D}$ contour is described by a function of $v$. The contour is placed on an image as $\boldsymbol{f}: \mathbb{R}^{\mathbf{3}} \rightarrow \mathbb{R}$. The snake model combines the internal energy $\boldsymbol{E}_{\text {int }}$ and external energy $\boldsymbol{E}_{\text {ext }}$ into $\boldsymbol{E}=\boldsymbol{E}_{\text {int }}(\boldsymbol{v})+\boldsymbol{E}_{\text {ext }}(\boldsymbol{v})$. The 3D image force concerns the movement in 3 directions of the parametric curve in $\boldsymbol{E}_{\text {int }}(\boldsymbol{v})$. Therefore, the parameter function $(\boldsymbol{v}=\boldsymbol{v}(\boldsymbol{s}, \boldsymbol{r})=[\boldsymbol{X}(\boldsymbol{s}, \boldsymbol{r}), \boldsymbol{Y}[\boldsymbol{s}, \boldsymbol{r}], \boldsymbol{Z}(\boldsymbol{s}, \boldsymbol{r})])$ is added to control the corresponding points (or the control points) where $\boldsymbol{X}, \boldsymbol{Y}, \boldsymbol{Z}$ are the corresponding coordinate function of the surface. The internal energy $\boldsymbol{E}_{\text {int }}(\boldsymbol{v})$ can be expressed as;

$$
E_{i n t}=\iint\left[\alpha_{s}\left|v_{s}^{\prime}\right|^{2}+\alpha_{r}\left|v_{r}^{\prime}\right|^{2}\right]+\left[\beta_{s}\left|v_{s s}^{\prime \prime}\right|^{2}+\beta_{r}\left|v_{r r}^{\prime \prime}\right|^{2}+\beta_{s r}\left|v_{s r}^{\prime \prime}\right|^{2}\right] d s d r
$$

where $\boldsymbol{\alpha}_{\boldsymbol{s}}$ and $\boldsymbol{\alpha}_{\boldsymbol{r}}$ denote the elasticity, respectively, $\boldsymbol{\beta}_{\boldsymbol{s}}$ and $\boldsymbol{\beta}_{\boldsymbol{r}}$ are the corresponding rigidities, and $\boldsymbol{\beta}_{\boldsymbol{s} \boldsymbol{r}}$ is the resistance to twist. 
http://wjst.wu.ac.th

The external energy $\boldsymbol{E}_{\text {ext }}(\boldsymbol{v})$ is the image force of the boundary. In this work, the EI model is modified from the 3D surface (EI model) to 3D matrix of contour point by setting the boundary of $\boldsymbol{I}_{\boldsymbol{E} I}(\boldsymbol{x}, \boldsymbol{y}, \boldsymbol{z})=\mathbf{1}$ and the inner and outer of a closed parametric surface (EE model) are set as 0 . The $\boldsymbol{E}_{\text {ext }}(\boldsymbol{v})$ represents by $\boldsymbol{E}_{\text {image }}(\boldsymbol{v}) . \boldsymbol{E}_{\text {image }}(\boldsymbol{v})$ shows the features of the EI model such as the boundary and represented it by potential force fields or the gradient of the image $\boldsymbol{\nabla} \boldsymbol{p}(\boldsymbol{v}, \boldsymbol{f})$ under the closed plane conditions. Next, the energy $\left(\boldsymbol{E}=\boldsymbol{E}_{\text {int }}(\boldsymbol{v})+\boldsymbol{E}_{\text {ext }}(\boldsymbol{v})\right)$ is minimized by using the Euler-Lagrange equation to find the $v$ that satisfies the equation balances the internal force and image force. When all energies are balanced, the total energy is minimum as shown.

$$
\left[\alpha_{s}\left|v_{s}^{\prime}\right|^{2}+\alpha_{r}\left|v_{r}^{\prime}\right|^{2}\right]-\left[\beta_{s}\left|v_{s s}^{\prime \prime}\right|^{2}+\beta_{r}\left|v_{r r}^{\prime \prime}\right|^{2}+\beta_{s r}\left|v_{s r}^{\prime \prime}\right|^{2}\right]=-\nabla p(v, f)
$$

The internal energy $\boldsymbol{E}_{\text {int }}(\boldsymbol{v})$ and its minimizing equation are set by using the 3D mesh of EE model. The characteristic of the internal force is to control the expanding motion of the parametric curve. The weighted parameters such as $\boldsymbol{\alpha}$ and $\boldsymbol{\beta}$ are used to control the tension and rigidity of the parametric curve. Figure 4 shows examples of when the energy is over controlled and cannot stop at the boundary.
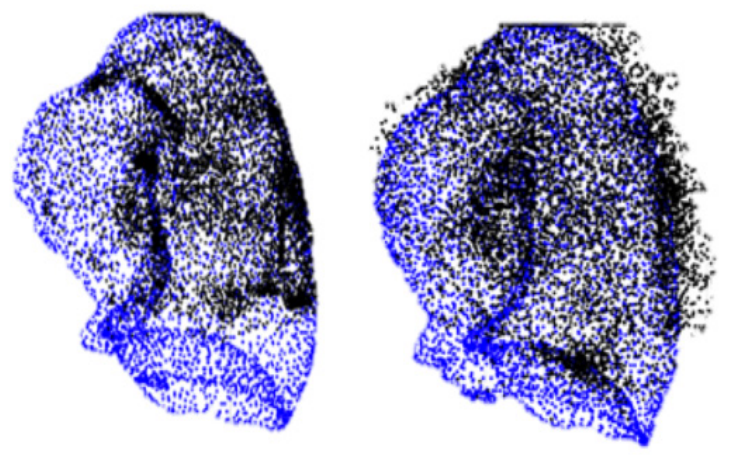

Figure 4 Examples when the magnitudes of weighted parameters are unbalanced.

To solve this problem as shown in Figure 4, the weighted parameters of the contour and image are estimated based on the conditions for each model calculated by $Z_{\text {ratio }}$ equation. The internal energy $\boldsymbol{E}_{\text {int }}(\boldsymbol{v})$ discourages stretching and bending of the contour while the image potential force pulls, pushing the contour toward the desired image boundary. The effect of shape and size of lung is used the adaptive parameter technique to find the suitable parameter. Due to the complexity of the parametric contour and the potential force fields, the parameters are allowed to adapt, depending on the individual shape of a lung as shown in Figure 5. Therefore, the additional step before applying 3D ACM is to measure the diameter of the lung in $\boldsymbol{x}, \boldsymbol{y}, \boldsymbol{z}$ directions. The $\boldsymbol{Z}_{\text {ratio }}$ is introduced to distinguish the different sets of shape (represented by range) estimated by the ratio of diameter EI to EE on the Z-axis as shown in $Z_{\text {ratio }}$ equation. The shape and size of lung is separated in to 3 sets based on the data set of this study and then

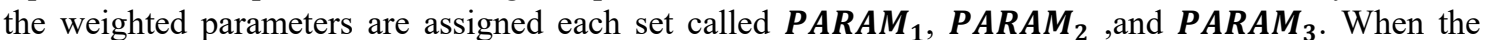
$\boldsymbol{Z}_{\text {ratio }}$ is higher than 1.2, $\boldsymbol{P A R A M _ { 1 }}$ is applied, the challenge of $\boldsymbol{P A R A} \boldsymbol{M}_{1}$ is the sharp edge in the lower lung, the balloon force and GVF are tuned. $P \boldsymbol{A R A} \boldsymbol{M}_{2}$ represents the normal or slightly abnormal lungs where $\boldsymbol{Z}_{\text {ratio }}$ is between 1 to 1.2. $\boldsymbol{P A R A} \boldsymbol{M}_{3}$ is the abnormality cases where mostly appears in patient with air trapping problem (obstructive lung disease). Figure 5 also demonstrates the $3 \mathrm{D}$ models at 3 different ranges of diameter. The $\boldsymbol{P A R A M _ { s }}$ consists of the weight of the image edge energy, image force, Gradient Vector Flow, and snake energies. They control the expanding and stop conditions of the deformable model from reaching the outer boundary. 
http://wjst.wu.ac.th

$Z_{\text {ratio }}$ is estimated by;

$$
Z_{\text {ratio }}=\frac{|\max (E I . z)-\min (E I . z)|}{|\max (E E . z)-\min (E E . z)|}
$$

where $|\max (\boldsymbol{E} \boldsymbol{I} . \mathbf{z})-\min (\boldsymbol{E} \boldsymbol{I} . \mathbf{z})|$ represents the diameter in Z direction of EI model and $\mid \max (\boldsymbol{E} \boldsymbol{E} . \mathbf{z})-$ $\min (\boldsymbol{E} E . z) \mid$ is the diameter in $Z$ direction of EE model. The weighted parameter function depends on the range of $\boldsymbol{Z}_{\text {ratio }}$ as explained below:

$$
Z_{\text {ratio }}=\left\{\begin{array}{lr}
\text { PARAM }_{1}, & x \geq 1.2 \\
\text { PARAM }_{2}, & 1<x<1.2 \\
\text { PARAM }_{3}, & x \leq 1
\end{array}\right.
$$

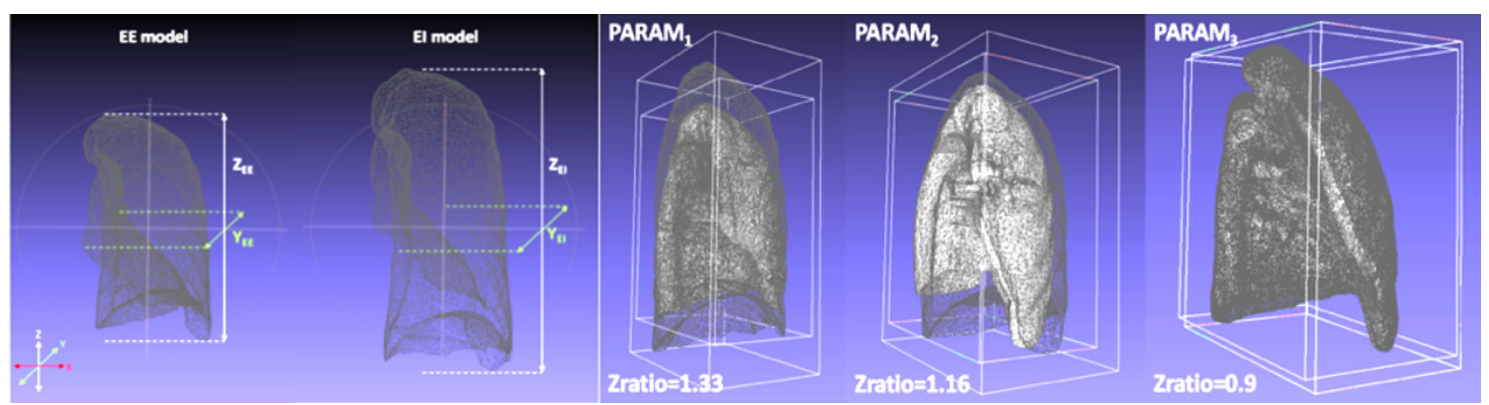

Figure $5 \boldsymbol{Z}_{\text {ratio }}$ estimation from EI and EE models and examples of a dataset with 3 different parameter function: $\boldsymbol{P A R A M} M_{1}, \boldsymbol{P A R A M}_{2}$, and $\boldsymbol{P A R A M _ { 3 }}$.

The Balloon Force and Gradient Vector Flow (GVF) are mainly adjusted because these two forces help control the surface to reach the outer boundary and supervise the inflation force to be stopped by the outer edges. The velocity vector is measured from the corresponding points on the EE model, represented by 3D mesh format, called FV model. The structure of $\boldsymbol{F} \boldsymbol{V}$ contains $\boldsymbol{F} \boldsymbol{V}$. faces with a facelist $(\boldsymbol{N} \times$ 3) and $\boldsymbol{F V}$. vertices with an $(\boldsymbol{N} \times \mathbf{3})$ vertex list. The velocity vector map is generated by the accumulation of motion of the parametric contour as shown:

$$
d\left(x^{\prime}, y^{\prime}, z^{\prime}: x, y, z\right)=\sqrt{\left(x^{\prime}-x\right)^{2}+\left(y^{\prime}-y\right)^{2}+\left(z^{\prime}-z\right)^{2}}
$$

The magnitude and direction from FV.vertices to FV.vertices' is a velocity vector where FV.vertices' is the stopping point (EI phase) and FV.vertices' is the starting point (EE phase). In this research, $\boldsymbol{d}(\Delta \boldsymbol{F V}$. vertices $)$ is used to analyze the inhomogeneous motion pattern of lung. The velocity vector map calculated by $\boldsymbol{d}(\Delta \boldsymbol{F V}$. vertices $)$ is managed as a feature of the classification model.

The human lungs are divided into 5 distinct anatomic compartments called lobes which are separated by the pulmonary fissures. The lung lobe separation is applied to identify the characteristics that predominate in each lobe. Point-based registration of oblique fissures and horizontal fissures are based on anatomical characteristics of an individual lung. It is shown in the literature [13] that fissures appear naturally as a 3D surface separating adjacent lung lobes. Then, they combined the segmentation in 2D and $3 \mathrm{D}$ as a hybrid approach to fill in an incomplete and disrupted fissure automatically. However, in this study, the approximate cutting plane of each lobe is used. The left lung has the upper $(\boldsymbol{L} \boldsymbol{U} \boldsymbol{L})$ and lower 
http://wjst.wu.ac.th

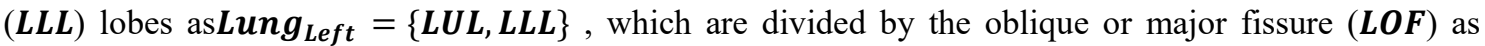
Oblique fissure $_{\text {left }}=\operatorname{LOF}\left(L_{O F 1}, L_{O F 2}, L_{O F 3}\right)$.

The right lung has the upper $(\boldsymbol{R} \boldsymbol{U} \boldsymbol{L})$, middle $(\boldsymbol{R} \boldsymbol{M} \boldsymbol{L})$, and lower $(\boldsymbol{R} \boldsymbol{L} \boldsymbol{L})$ lobes as Lung $_{\text {Right }}=\{$ RUL, RML, RLL $\}$. The upper and middle lobes are separated by the horizontal or minor fissure $(\boldsymbol{R H} \boldsymbol{F})$; both upper and middle lobes are separated from the lower lobe by the right oblique (major) fissure $(\boldsymbol{R O F})$ as shown:

$$
\text { Oblique fissure } \text { right }=\operatorname{RHF}\left(R_{H F 1}, R_{H F 2}, R_{H F 3}\right), \operatorname{ROF}\left(R_{O F 1}, R_{O F 2}, R_{O F 3}\right)
$$

The cutting plane is generated and then the cuboid is created to select the region of interest (ROI). To localize the cutting plane, there are three landmark points as explained above. To create the plane, the normal vector $\overrightarrow{\boldsymbol{n}}=[\boldsymbol{a}, \boldsymbol{b}, \boldsymbol{c}]$ needs to be determined by crossing two vectors on the plane $(\boldsymbol{a} \boldsymbol{x}+\boldsymbol{b} \boldsymbol{y}+$ $\boldsymbol{c z}+\boldsymbol{d}=\mathbf{0}$ ). After we obtain the cutting plane, the angle of rotation can be calculated. For the process of the angle of rotation [14], it is found that on sagittal views, the oblique and horizontal fissures are oriented at approximately 45 degrees and 90 degrees, respectively, with respect to the $Z$-axis. The point cloud data is rotated, based on the degree of rotation, to select the ROI.

For the lung lobe separation of the left lung, the step to generate the cutting plane and cuboid ROI of the left lung. Right lung separation is complicated than the left lung separation because it consists of two fissures: RHF and ROF. Firstly, the ROF is performed by cutting RLL out of RUL and RML. Secondly, the RHF is made and cuts the RUL and RML.

\section{Feature analysis and classification}

The velocity vector map calculated by $\boldsymbol{d}(\Delta \boldsymbol{F V}$. vertices $)$ is used to analyze the expanding motion of lung. After applying lung lope separation, there are 5 sets of lung lopes: $\boldsymbol{L u n g}_{\text {Left }}=\{\boldsymbol{L U} \boldsymbol{L}, \boldsymbol{L L L}\}$ and $\boldsymbol{L u n g}_{\text {Right }}=\{\boldsymbol{R} \boldsymbol{U} \boldsymbol{L}, \boldsymbol{R} M \boldsymbol{L}, \boldsymbol{R} \boldsymbol{L} \boldsymbol{L}\}$. The complex features are simplified by using bag-of-words (BOW) model to represent in the same dimension. The velocity vector with a different number of control points is applied to the BOW model to simplify the dimensional information into an accumulated histogram represented by $\boldsymbol{d}(\Delta \boldsymbol{F V}$.vertices $)$. The vector set of lung lobes are obtained called visual velocity vectors. After that the normalization $\left(\boldsymbol{x}_{i}^{\prime}=\left(\frac{x_{i}-x_{\min }}{x_{\max }-x_{\min }}\right)\left(\boldsymbol{x}_{\max }^{\prime}-\right.\right.$ $\left.\boldsymbol{x}_{\text {min }}^{\prime}\right)+\boldsymbol{x}_{\text {min }}^{\prime}$ ) is applied to each visual velocity vectors in order to deal with distribution of data where $\boldsymbol{x}_{\boldsymbol{i}}^{\prime}$ is the actual input feature, $\boldsymbol{x}_{\min }$ and $\boldsymbol{x}_{\max }$ are the minimum and maximum value of input feature and $\boldsymbol{x}_{\text {min }}^{\prime}$ and $\boldsymbol{x}_{\text {max }}^{\prime}$ are the minimum and maximum target range of input feature which is [0,1]. Next, the machine learning techniques are: Artificial Neural Network (ANN), and Support Vector Machine (SVM).

In the ANN model, the number of hidden layers $\left(\mathrm{N}_{\mathrm{L}}\right)$ is set as 20 based on the $\boldsymbol{N}_{\boldsymbol{i}}$ and $\boldsymbol{N}_{\boldsymbol{o}}$. For the number of hidden neurons $\left(\boldsymbol{N}_{\boldsymbol{N}}\right)$, there are many rules of thumb for calculating $\boldsymbol{N}_{\boldsymbol{L}}$ such as method [17] $\boldsymbol{N}_{\boldsymbol{n}}=\sqrt{\boldsymbol{N}_{\boldsymbol{i}} \boldsymbol{N}_{\boldsymbol{o}}}$ where the input neuron and output neuron represent by $\boldsymbol{N}_{\boldsymbol{i}}$ and $\boldsymbol{N}_{\boldsymbol{o}}$, method [16], $\boldsymbol{N}_{\boldsymbol{n}}=$ $2^{N_{i}}-\mathbf{1}$, method [15], $N_{h}=\frac{2^{N_{i}}}{N_{i}}+\mathbf{1}$. In this research, we optimize the performance and $N_{n}=\sqrt{N_{i} N_{o}}+$ $\mathbf{1}$ is used for setting the hidden neurons. The activation function of output layer is TanH function $\left(\boldsymbol{T a n H}=\frac{2}{1+e^{-2 x}}-\mathbf{1}\right)$ which has range values between $(0,1)$. The SVM model with Radial Basis Function (RBF) kernel function $\boldsymbol{K}\left(\boldsymbol{x}, \boldsymbol{x}^{\prime}\right)=\exp \left(-\gamma_{\boldsymbol{R} B \boldsymbol{F}}\left\|\boldsymbol{x}-\boldsymbol{x}^{\prime}\right\|^{2}\right)$ is selected. The $\left\|\boldsymbol{x}-\boldsymbol{x}^{\prime}\right\|^{2}$ is the squared Euclidean distance between 2 feature vectors. The gamma value $\left(\gamma_{R B F}\right)$ of the RBF kernel function is equal to 12. The $\gamma_{\boldsymbol{R} B F}$ is the invert of standard deviation of the Gaussian function $\gamma_{\boldsymbol{R} B F}=\frac{\mathbf{1}}{\mathbf{2 \sigma ^ { 2 }}}$ . If $\gamma_{R B F}$ is too small, the hyper plane is almost flat and when the gamma is too high, the variance of distribution is small.

To predict the output in supervised machine learning (ML), the labels are set at 0 and $1(0=$ healthy and 1 = abnormal). The result is explained by the confusion matrix, and the performance of the 
http://wjst.wu.ac.th

classification process is evaluated using 10 -fold cross-validation by separating into 10 folds with $10 \%$ for testing and $90 \%$ for training. The predictive model is separated into two sub-modules. The first module classifies the normal and abnormal cases by using the $\boldsymbol{Z}_{\text {ratio }}$ and $\boldsymbol{Y}_{\text {ratio }}$, combined with the motion feature of each lung lobe. Secondly, lung diseases are divided into obstructive and restrictive a pattern using Module 2. To separate the obstructive and restrictive patterns, the inspiratory and expiratory phases of healthy people are used to analyze the similarity of abilities to expand and shrink, based on the relevant knowledge of PFTs, FVC, FEV1, TLC, and $\mathrm{FEV}_{1} / \mathrm{FVC}$ ratio.

\section{Results and discussion}

The confusion matrix is used to evaluate the performance of the predictive model by accumulating the results from Module 1 and Module 2. The Weighted Average Precision $\left(P R E_{A v g}\right)$ is used to overcome the unbalanced data problem. To interpret, the confusion matrix in medical use is slightly different from the usual situation. The definition of each element needs to be clarified as follows: True Positive (TP) is when the patient has a lung disease, and the model correctly predicts, True Negative (TN) is when the patient is healthy, and the model correctly predicts, False Positive (FP) is when the patient is healthy, but the model predicts as lung disease, False Negative (FN) is the patient has a lung disease, but the model cannot detect and predicts as healthy. All measurement terms are used to analyze the effectiveness of the model from sensitivity (SENS), specificity (SPEC), and precision (PRE). The sensitivity (SENS) is the rate of TP over all of actual class $\left(N_{\text {actual_lung }}\right)$ where $N_{\text {actual_lung }}$ calculated by $N_{\text {actual_Lung }}=T P+$ $F N$. The $S E N S$ formula is $S E N S=\frac{T P}{T P+F N}$. The specificity $(S P E C)$ is the rate of TN over all of actual class of healthy ( $N_{\text {actual_healthy }}$ ) where $N_{\text {actual_healthy }}=T N+F P$. It is defined as $C=\frac{T N}{T N+F P}$. The precision $(P R E)$ is the rate of TP over all positive events. It is defined $P R E=\frac{T P}{T P+F P}$. Due to the unbalanceddata, the performance measure is used in the weighted average technique. Weighted Average Precision $\left(P R E_{A v g}\right)$ is used as $P R E_{A v g}=\frac{w_{i-1} \times P R E C_{i-1}+w_{i} \times P R E C_{i}}{w_{i-1}+w_{i}}$, where $w_{i}$ represents the number of data points in each class, where $i$ is class number.

\section{Classification results}

The classification model consists of predictive hierarchy modules (2 layers). Module 1 classifies normal and abnormal lungs. Module 2 separates obstructive and restrictive lung patterns. Evaluation of Module 1: Classify normal and abnormal respiratory patterns of lungs. The total number of data is 120 sets which 80 sets are abnormal, and 40 sets are normal. The K-fold cross-validation is applied to separate training and testing sets. The selected features are the histogram of the velocity vectors for each lobe, $Y_{\text {ratio }}$, and $Z_{\text {ratio }}$. The stepwise regression is applied to reduce the feature dimensions and then the ANN and SVM are selected as classifier techniques to analyze the normal and abnormal cases. The PRE $E_{\text {normal }}$ and $\mathrm{PRE}_{\text {abnormal }}$ are calculated for normal and abnormal classes. Due to the unbalanced data, the PRE $\mathrm{AVG}_{\mathrm{AV}}$ is evaluated to find the performance for each model. They show that $\mathrm{PRE}_{\mathrm{Avg}}$ of module 1 is $84.07 \%$ with ANN, and $85.66 \%$ with SVM technique. The FP is $8.33 \%$ and the FN is $5.83-7.5 \%$. The SENSs of ANN and SVM are 88.75 and $91.25 \%$, respectively and the SPEC is $75 \%$.

Evaluation of Module 2: Two different types of lung disease are classified by learning the pattern of inspiratory from the normal data, the histogram of the velocity vectors for each lobe, $Y_{\text {ratio }}$, and $Z_{\text {ratio }}$. Based on the hierarchy predictive model, the total data for Module 2 is 80 sets with 40 obstructive and 40 restrictive patterns. The $K$-fold cross-validation and stepwise regression are applied and then ANN and SVM is used to compare the performance of the module as shown in Table 2. They show that the PRE Avg of ANN is $87.91 \%$ and $\mathrm{PRE}_{\mathrm{Avg}}$ of $\mathrm{SVM}$ is $82.34 \%$. The confusion matrix of Module 2 is changed by setting TP is a restrictive pattern and the model predicts correctly, TN is an obstructive pattern and the model predicts correctly, FP is obstructive pattern, but the model predicts as restrictive pattern, and FN is a restrictive pattern, but the model detects an obstructive pattern. From Table 2, Module 2 of ANN and SVM has the FP at $7.5-8.75 \%$ and the FN at $10-12.5 \%$. The SENSs of ANN and SVM are 75 and 80 $\%$ and the SPECs are 82.4 and $84.62 \%$, respectively. 
http://wjst.wu.ac.th

Table 2 Evaluation results of Module 1 ( $\mathrm{PRE}_{0}$ represents normal and $\mathrm{PRE}_{0}$ represents abnormal) and Module 2 ( $\mathrm{PRE}_{0}$ represents obstructive and $\mathrm{PRE}_{1}$ represents, restrictive).

\begin{tabular}{lllllllll}
\hline Module & Models & \multicolumn{9}{c}{ Confusion Matrix } & \multicolumn{3}{c}{ Evaluation } \\
\cline { 2 - 9 } & & TP & TN & FP & FN & PRE $_{0}$ & PRE $_{1}$ & PRE $_{\text {AVG }}$ \\
\hline Module 1 & ANN & 71 & 30 & 10 & 9 & 87.65 & 76.92 & 84.07 \\
\cline { 2 - 9 } & SVM & 73 & 30 & 10 & 7 & 87.95 & 81.08 & 85.66 \\
\hline \multirow{2}{*}{ Module 2 } & ANN & 30 & 33 & 7 & 10 & 81.08 & 76.74 & 78.91 \\
& SVM & 32 & 33 & 6 & 8 & 84.20 & 80.48 & 82.34 \\
\hline
\end{tabular}

In addition, Module 2 is related to the pathological knowledge of restrictive and obstructive lung diseases. The restrictive lung disease happens when the patient cannot take a full and deep inhalation. Therefore, when comparing the normal size of a lung in EI CT images, a patient with restrictive lung disease can be differentiated from a normal patient. In the obstructive lung disease, the patient has a problem with exhalation. Patients cannot exhale naturally because there is a blocked area in the small airway or bronchus. The predictive model that is used to classify obstructive lung diseases is the EE CT images of a normal patient and an obstructive lung disease patient. It is found that the restrictive pattern has more false predictions than the obstructive pattern. However, the limitations of the classification are the variety of disease severities.

\section{Conclusions}

This work introduces a new motion detection of the lung using a velocity vector map-generating from the 3D ACM technique and studies inhomogeneous motion patterns from each lung lobe to generate predictive models for the automatic screening of lung disease. The velocity vectors of the EI and EE models are evaluated by the corresponding points on the parametric surface model of the EE model to the EI model. In the 3D ACM, the external energy from the EI model is the external force that pushes the 3D parametric surface to reach the boundary. The external forces, such as the balloon force and GVF, are adjusted adaptively by the shape conditions $\left(Z_{\text {ratio }}\right)$ which is calculated from the ratio of the diameter from $E I$ to $E E$ on the $Z$ axis. Next, the feature representation is studied and evaluated based on the lung structure, separated into five lobes. ANN and SVM techniques are applied to classify those patterns to screen the lung diseases into normal, obstructive, and restrictive patterns.

In conclusion, the inhomogeneous motion patterns of lungs combined with medical-based knowledge can be used to analyze lung diseases. However, the limitations of this work are the amount of data and the level of severity of diseases. Therefore, misclassification may occur in the early stages of lung diseases which motion patterns cannot observe. For future works, we plan to increase the amount of data and the variety of the severity of diseases to improve the performance of the classification model. We also plan to increase the accuracy by finding more relative points of EE and EI inhomogeneous motion, studying feature representation and feature selection, and increasing the number of databases.

\section{Acknowledgements}

This research is supported by a dual-degree scholarship from the Japan Advanced Institute of Science and Technology (JAIST), Sirindhorn International Institute of Technology (SIIT), and National Science and Technology Development Agency (NSTDA), and the Center of Excellence in Biomedical Engineering, Thammasat University. This work also was financially supported by Thammasat University (TU), a TU research grant [grant numbers TU2016015]. The authors would also like to express our thanks to the Department of Radiology, Faculty of Medicine, Thammasat University, and Kanazawa hospital for supporting us with medical advice and a dataset. 
http://wjst.wu.ac.th

\section{References}

[1] World Health Organization. The top 10 causes of death. World Health Organization. Available at: https://www.who.int, accessed June 2020.

[2] SF Nemec, AA Bankier and RL Eisenberg. Upper lube-predominant diseases of the lung. Amer. J. Roentgenol. 2013; 200, W222-W237.

[3] JD Backer. FRI and disease characterization: Idiopathic pulmonary fibrosis. Fluidda, 2018.

[4] AJ Blaivas and W Strauss. Middle Lobe Syndrome in the Left Lower Lobe in Chronic Obstructive Pulmonary Disease. Prim. Care Respir. J. 2019; 18, 331-3.

[5] SF Nemec, AA Bankier and RL Eisenberg. Lower lube: Predominant diseases of the lung. Amer. J. Roentgenol. 2013; 200, 712-28.

[6] Y Qu, Y Cao, M Liao and Z Lu. Sagittal-lung CT measurements in the evaluation of asthma-COPD overlap syndrome: A distinctive phenotype from COPD alone. J. Italian Soc. Med. Radiol. 2017; 122, 487-97.

[7] RA Hartley, BL Barker, C Newby, M Pakkal, S Baldi, R Kajekar, R Kay, M Laurencin, RP Marshall, AR Sousa, H Parmar, S Siddiqui, S Gupta and CE Brightling. Relationship between lung function and quantitative computed tomographic parameters of airway remodeling, air trapping, and emphysema in patients with asthma and chronic obstructive pulmonary disease: A single-center study. J. Allergy Clin. Immunol. 2016; 137, 5.

[8] M Zehtabian, R Faghihi, MA Mosleh-Shirazi, AR Shakibafard, M Mohammadi and M BaradaranGhahfarokhi. A fast model for prediction of respiratory lung motion for image-guided radiotherapy: A feasibility study, Iran. J. Radiat. Res. 2012; 10, 73-81.

[9] H Ladjal, N Skendraoui, M Giroux, Y Touileb, J Azencot, B Shariat, H Ladjal, M Beuve and P Giraud. Physiology and biomechanical model of patient specific lung motion based on 4D CT images. In: Proceedings of the 2015 Biomedical Engineering International Conference. Pattaya, Thailand, 2015, p. 1-5.

[10] L Han, H Dong, JR McClelland, L Han, DJ Hawkes and DC Barratt. A hybrid patient-specific biomechanical model based image registration method for the motion estimation of lungs. Med. Image Anal. 2017; 29, 87-100.

[11] CP Hersh, GR Washko and RSJ Estepar. Paired inspiratory-expiratory chest CT scans to assess for small airways disease in COPD. Respir. Res. 2013; 14, 42.

[12] B Fuerst, T Mansi, F Carnis, M Salzle, J Zhang, J Declerck, T Boettger, J Bayouth, N Navab and A Kamen. Patient-specific biomedical model for the prediction of lung motion from 4-D CT images. IEEE Trans. Med. Imag. 2015; 34, 2.

[13] Q Wei and Y Hu. A fybrid approach to segmentation of diseases lung lobes. IEEE J. Biomed. Health Inform. 2014; 18,3.

[14] S Ukil and JM Reinhardt. Anatomy-guided lung lobe segmentation in X-ray CT images. IEEE Trans. Med. Imag. 2009; 28, 2.

[15] Z Zhang, X Ma and Y Yang. Bounds on the number of hidden neurons in three-layer binary neural networks. Neural Netw. 2003; 16, 995-1002.

[16] D Hunter, H Yu, MS Pukish III, J Kolbusz and BM Wilamowski. Selection of proper neural network sizes and architectures: A comparative study. IEEE Trans. Ind. Inform. 2012; 8, 228-40.

[17] K Shibata and Y Ikeda. Effect of number of hidden neurons on learning in large-scale layered neural networks. In: Proceedings of the ICROS-SICE International Joint Conference 2009. Fukuoka, Japan, 2009, p. 5008-13.

[18] PP Walker, P Mitchell, F Diamantea, CJ Warburton and L Davies. Effect of primary-care spirometer on the diagnosis and management COPD. Eur. Respir. J. 2006; 28, 945-52. 